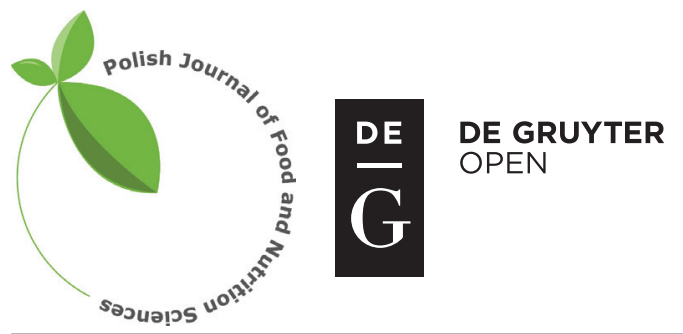

Pol. J. Food Nutr. Sci., 2018, Vol. 68, No. 1, pp. 5-13 DOI: $10.1515 /$ pjfns-2017-0012 http://journal.pan.olsztyn.pl

Original research article

Section: Food Technology

\title{
Effect of Blanching Techniques and Treatments on Nutritional Quality of Dried Mango Slices During Storage
}

\author{
Asad Gulzar', Mahmood Ahmed ${ }^{2 *}$, Muhammad Abdul Qadir ${ }^{2}$, Muhammad Imtiaz \\ Shafiq ${ }^{3}$, Sakhawat Ali', Ijaz Ahmad ${ }^{4}$, Muhammad Farooq Mukhtar ${ }^{1}$ \\ ${ }^{1}$ Division of Science and Technology, University of Education, Lahore, Pakistan \\ ${ }^{2}$ Institute of Chemistry, University of the Punjab, 54590 Lahore, Pakistan \\ ${ }^{3}$ Institute of Biochemistry and Biotechnology, University of the Punjab, 54590 Lahore, Pakistan \\ ${ }^{4}$ Food and Biotechnology Lab, PCSIR Laboratories, Lahore, Pakistan
}

Key words: infrared, microwave, blanching, osmotic dehydration, enzyme inactivity

The present invention was undertaken to study and determine the effect of potassium metabisulphite (6\%) and potassium sorbate (350 ppm) treatments on the nutritional quality of osmotically-dehydrated, infrared- and microwave-blanched dried mango slices (local cultivars "Chaunsa" and "Fajri") stored for the period of 6 months under ambient conditions. The studied parameters included physical characteristics such as water activity, non-enzymatic browning, and color values, chemical parameters such as moisture, ash, fiber, acidity and content of proteins, sugars, vitamin C, total carotenoids, and sensory attributes such as appearance, flavor and texture. Vitamin C content in osmotically-dried mango slices was higher than that of IR and MW blanched dried mango slices but the content of vitamin C of both cultivars was lower than of the fresh mango samples (Chaunsa: $135 \mathrm{mg} / 100 \mathrm{~g}$, Fajri: $94 \mathrm{mg} / 100 \mathrm{~g}$ ). Significant loss was noticed in total carotenoids content of both the cultivars with passage of time because of their susceptibility to oxidative loss caused by dry heat. No growth of yeast and mold was detected in potassium sorbate-treated dried mango slices due to their preservative effect. From the point of view of the composition and sensory quality, dried mango slices of both the cultivars have excellent nutritional qualities.

\section{INTRODUCTION}

Mango (Mangifera indica L.) is one of the most important tropical fruits having a distinct and attractive color and aroma that has been commercialized worldwide in fresh or processed form. The global production of mango is accounting for nearly $50 \%$ of world tropical fruit production [Singh et al., 2000; Slavin \& Lloyd, 2012]. Mango is a rich source of many nutrients and bioactive compounds like: carotenoids, vitamin C, phenolics, dietary fiber, carbohydrates, and minerals such as potassium, iron and calcium. It has been of interest and essential to human health and nutrition to prevent many deceases like cardiovascular and neurogenerative diseases and cancer (respiratory system, lungs). Fiber is important for normal functioning of the gastrointestinal tract; also it lowers the risk of cardiovascular and coronary heart diseases as well as maintains the normal body weight [Hopping et al., 2010; Lemmens et al., 2013; Rincon \& Kerr, 2010; Slavin, 2013; Sogi et al., 2012].

Shelf-life enhancement and reduction of postharvest loses of fruits is usually done by the air drying preservation method. However, the quality of dried products, such as color,

\footnotetext{
* Corresponding Author: Tel.: +923008819844, +923334878610

E-mail: mahmoodresearchscholar@gmail.com (Dr. M. Ahmed)
}

flavor, texture, rehydration capacity and nutrient contents, are negatively affected by long drying time or high temperature. Carotenoids and vitamin $\mathrm{C}$ are most sensitive compounds to heating procedures. Prior to air drying of fruits, pretreatment such as blanching and osmotic dehydration (OD) are commonly used to preserve the quality of fruits [Ghosh et al., 2012; Guiamba \& Svanberg, 2016]. Besides the advantages of OD, the leaching of water soluble components such as vitamins, minerals and organic acids may be extensive during OD and it can also be detrimental to sensory and nutritional values of dried fruits [Chandra \& Kumari, 2015; Yadav \& Singh, 2014]. The method of blanching is frequently used in fruit processing to avoid enzymatic reactions by polyphenol oxidase (PPO) and ascorbic acid oxidase (AAO) enzymes. Through the browning reaction, PPO affects the appearance and organoleptic properties of fruits by oxidation of phenolic compounds to quinones while AAO converts L-ascorbic acid (LAA) to dehydroascorbic acid (DHAA) by oxidation, subsequently inducing the loss of vitamin C. Dry blanching such as infrared (IR) and microwave (MW) electromagnetic radiation is emerging to process the fruits as an alternative to the conventional water blanching to minimize the loss of water-soluble compounds and it also improves the nutritional value of processed fruits [Holzwarth et al., 2013; Ruiz-Ojeda \& Peñas, 2013; Vishwanathan et al., 2013]. En- 
zymatic and non-enzymatic reactions cause the browning of fruits and subsequently their quality deterioration. Antibrowning agents have been successfully investigated to overcome the problem induced by enzymatic and non-enzymatic browning. Inhibition of enzymatic and non-enzymatic browning can also be achieved by sulphites and sorbates like potassium metabisulphite and potassium sorbate [Lemmens et al., 2013; Munyaka et al., 2010; Sra et al., 2014]. Hence, the present study aimed to evaluate the influence of blanching techniques and treatment of mango slices with potassium metabisulphite and potassium sorbates on their nutritional quality after drying and storage.

\section{MATERIALS AND METHODS}

\section{Sample collection and preparation}

Two mango cultivars (local names "Chaunsa and Fajri") were bought from shelves in the capital city Lahore $\left(31^{\circ} 33^{\prime} 16.58^{\prime \prime} \mathrm{N}\right.$, 74²1'25.77”E), Punjab, Pakistan. 24 kg of Fajri (FJR) mangoes and $16 \mathrm{~kg}$ of Chaunsa (CHS) mangoes were washed, manuallypeeled and cut into $6-8 \mathrm{~cm}$ long and $2 \mathrm{~cm}$ thick mango slices. $14.4 \mathrm{~kg}$ of FJR and $8 \mathrm{~kg}$ of CHS mango slices were distributed into 3 lots separately.

\section{Blanching procedures and treatment of blanched slices}

Mango slices were blanched using near infrared radiation (IR) or microwaves (MW). The LEQUIP IR D5 dehydrator (China) was used for IR blanching. IR output power systematically changed and power sequence to maintain the temperature of $90^{\circ} \mathrm{C}$ for $2 \mathrm{~min}$ was as follows: IR output $100 \%$, IR heat reflux $14.3 \mathrm{~kW} / \mathrm{m}^{2}$, blanching time $72 \mathrm{~s}$, and IR output $30 \%$, IR heat reflux $4.4 \mathrm{~kW} / \mathrm{m}^{2}$, blanching time $48 \mathrm{~s}$. Microwave (MW) blanching using Microwave lab station (Milestones, Italy) was conducted at $1350 \mathrm{~W} / 120$ s to maintain the temperature of $90^{\circ} \mathrm{C}$ for $2 \mathrm{~min}$ [Eliasson et al., 2014; Guiamba \& Svanberg, 2016]. Afterwards, the blanched slices were dipped in $6 \%$ potassium metabisulphite (KMS) for 40 min and in a $350 \mathrm{ppm}$ solution of potassium sorbates (PS) for $10 \mathrm{~min}$. The samples not dipped in KMS and PS were considered the control samples. The ratio of mango slices to the dipping solution was 1:2. The slices were further dried at $60 \pm 5^{\circ} \mathrm{C}$ for $7 \mathrm{~h}$ in a hot air oven (Vision Scientific, Korea) to a constant weight for final product preparation. In addition, new portion of mango slices was immersed in KMS or PS (without IR and MW blanching) and osmotically dehydrated (OD). A sugar solution ( $45^{\circ}$ Brix) was prepared by dissolving the refined sugar purchased from the local market of Lahore and refractive index (Fisher Scientific ${ }^{\mathrm{TM}}$ Refractometer, UK) was measured. The samples were dehydrated osmotically at $25^{\circ} \mathrm{C}$ for $15 \mathrm{~h}$ then hot air dried at $50^{\circ} \mathrm{C}$ for $5 \mathrm{~h}$.

\section{Packaging and storage}

High density polyethylene (HDPE) having $60 \mu \mathrm{m}$ thicknesses was used to pack the dried mango slices, that were then sealed with the help of an impulse heat sealer. Each package contained $100 \mathrm{~g}$ of dried slices and all packages were stored in an environmental test chamber (Sanyo, Japan) at $27 \pm 2{ }^{\circ} \mathrm{C}$ temperature and $55 \pm 10 \%$ relative humidity for the period of 6 months.

\section{Physiochemical and microbiological analysis}

The dried mango slices were analysed for moisture, ash and fiber contents, acidity, sugars (total, reducing and nonreducing sugars) ascorbic acid, protein, non-enzymatic browning (as optical density) and total carotenoids by AOAC methods and methods reported earlier [Ahmad et al., 2016; Ahmed et al., 2016a, b; Ranganna, 1986]. Chilled mirror dew point technique was employed to measure the water activity $\left(\mathrm{a}_{\mathrm{w}}\right.$ ) by an activity meter (Aqualab CX-3 TE, USA) while the color was measured by lab color difference meter (Colour Meter PCE-TCR 200, UK). Potassium sorbates and $\mathrm{SO}_{2}$ were also measured by the earlier reported methods [Ahmed et al., 2013; Sra et al., 2014]. Total plate count, yeast and mold count in dried mango slices were also investigated [Banwart, 2012].

\section{Sensory evaluation}

To judge the acceptability and sensory qualities of the product, the dried mango slices were presented to a trained panel of seven judges ( 4 males and 3 females) to evaluate a total of 13 sensory attributes: 3 attributes of appearance like skin, pulp colors and evenness of color; 6 attributes of flavor like bitterness, fruity, sweetness, burnt, woody, after taste; and 4 attributes of texture such as skin residues, juiciness, hardness and tooth-packing. The 0-10 numerical scale with increments of 1 was used to quantify the intensity of attributes, where 0 represents none and 10 extremely strong according to the method described by Meilgaard et al. [2006].

\section{Statistical analysis}

SPSS version 15 (SPSS Inc., Chicago, IL, USA) for Windows was used for the statistical analysis; the main effects viz. treatments, blanching (fixed factor) and months (random factor) were evaluated by using the analysis of variance (ANOVA). Means and interactions were determined by general linear models $(\mathrm{GLM})$ at a $95 \%$ level of significance $(\mathrm{P}<0.05)$ for each cultivar respectively and post hoc test was performed for multiple comparisons.

\section{RESULTS AND DISCUSSION}

\section{Physiochemical properties}

The results of $\mathrm{a}_{\mathrm{w}}$ are presented in Table 1 at the initial stage ( 0 month) and after 3 and 6 months of storage. The $\mathrm{a}_{\mathrm{w}}$ of dried mango slices for both the cultivars increased significantly $(p<0.05)$ during the storage for 6 months. For CHS cultivar, no significant difference $(p<0.05)$ was observed among the blanching techniques and treatments as well as the interaction of blanching with treatment. For FJR cultivar, a significant difference $(p<0.05)$ was observed for factors (blanching technique, treatments, and storage) as well as for the interaction of blanching and storage and no significance different observed for the interaction of blanching technique with storage. The influential factor for food evaluation by consumers is its color in spite of good taste and flavor, off color of food products is often unacceptable. The effect of osmotic dehydration, IR and MW blanching on the color of dried mango slices was evaluated. The $\mathrm{L}$, $\mathrm{a}$ and $\mathrm{b}$ color values of dried mango slices are presented in Table 1. No significant effect among the blanching technique $(p<0.05)$ was 
TABLE 1. Effect of treatments and storage on the physiochemical properties of dried mango slices.

\begin{tabular}{|c|c|c|c|c|c|c|c|c|c|c|c|c|c|c|c|c|c|c|}
\hline \multirow{3}{*}{$\begin{array}{l}\text { Storage } \\
\text { period } \\
\text { (months) }\end{array}$} & \multicolumn{6}{|c|}{ Potassium metabisulphite $6 \%$ (KMS) } & \multicolumn{6}{|c|}{ Potassium sorbates 350 ppm (PS) } & \multicolumn{6}{|c|}{ Control } \\
\hline & \multicolumn{3}{|c|}{ CHS } & \multicolumn{3}{|c|}{ FJR } & \multicolumn{3}{|c|}{ CHS } & \multicolumn{3}{|c|}{ FJR } & \multicolumn{3}{|c|}{ CHS } & \multicolumn{3}{|c|}{ FJR } \\
\hline & OD & IR & MW & OD & IR & MW & OD & IR & MW & OD & IR & MW & OD & IR & MW & OD & IR & MW \\
\hline \multicolumn{19}{|c|}{ Water activity $\left(a_{w}\right)$} \\
\hline 0 & 0.65 & 0.61 & 0.5 & 0.68 & 0.64 & 0.69 & 0.64 & 0.62 & 0.61 & 0.66 & 0.64 & 0.62 & 0.64 & 0.60 & 0.59 & 0.67 & 0.63 & 0.61 \\
\hline 3 & 0.69 & 0.68 & 0.62 & 0.72 & 0.68 & 0.78 & 0.71 & 0.67 & 0.64 & 0.69 & 0.67 & 0.65 & 0.67 & 0.63 & 0.62 & 0.71 & 0.66 & 0.64 \\
\hline 6 & 0.72 & 0.71 & 0.69 & 0.81 & 0.79 & 0.89 & 0.79 & 0.73 & 0.71 & 0.74 & 0.72 & 0.71 & 0.71 & 0.68 & 0.67 & 0.76 & 0.69 & 0.69 \\
\hline Mean & 0.69 & 0.67 & 0.63 & 0.74 & 0.70 & 0.77 & 0.70 & 0.67 & 0.65 & 0.66 & 0.68 & 0.66 & 0.67 & 0.64 & 0.66 & 0.71 & 0.66 & 0.65 \\
\hline
\end{tabular}

Significance for CHS: $\mathrm{B}=0.064^{\mathrm{NS}}, \mathrm{T}=0.501^{\mathrm{NS}}, \mathrm{M}=0.021^{\mathrm{s}}, \mathrm{B} \times \mathrm{T}=0.222^{\mathrm{NS}}, \mathrm{B} \times \mathrm{M}=0.265^{\mathrm{NS}}, \mathrm{T} \times \mathrm{M}=0.078^{\mathrm{NS}}$

Significance for FJR: $\mathrm{B}=0.010^{\mathrm{s}}, \mathrm{T}=0.041^{\mathrm{s}}, \mathrm{M}=0.013^{\mathrm{s}}, \mathrm{B} \times \mathrm{T}=0.000^{\mathrm{s}}, \mathrm{B} \times \mathrm{M}=0.421^{\mathrm{NS}}, \mathrm{T} \times \mathrm{M}=0.002^{\mathrm{s}}$

\begin{tabular}{lllllllllllllllllll}
\hline \multicolumn{10}{c}{ Moisture $(\%)$} \\
\hline 0 & 8.7 & 8.6 & 8.6 & 12.1 & 12.0 & 12.1 & 8.6 & 8.6 & 8.5 & 12.1 & 12.1 & 12.1 & 8.7 & 8.4 & 8.5 & 12.1 & 12.0 & 12.1 \\
3 & 9.2 & 9.3 & 9.2 & 12.8 & 12.7 & 12.7 & 9.2 & 9.4 & 9.1 & 12.7 & 12.6 & 12.8 & 9.3 & 9.1 & 9.3 & 12.7 & 12.6 & 12.7 \\
6 & 9.8 & 9.9 & 9.7 & 13.1 & 13.0 & 13.0 & 9.9 & 9.9 & 9.8 & 13.0 & 13.1 & 13.1 & 9.9 & 9.7 & 9.8 & 13.1 & 13.0 & 13.1 \\
Mean & 9.2 & 9.3 & 9.2 & 12.7 & 12.6 & 12.6 & 9.2 & 9.3 & 9.1 & 12.6 & 12.6 & 12.7 & 9.3 & 9.1 & 9.2 & 12.6 & 12.5 & 12.6
\end{tabular}

Significance for CHS: $\mathrm{B}=0.148^{\mathrm{NS}}, \mathrm{T}=0.529^{\mathrm{Ns}}, \mathrm{M}=0.0001^{\mathrm{s}}, \mathrm{B} \times \mathrm{T}=0.005^{\mathrm{s}}, \mathrm{B} \times \mathrm{M}=0.219^{\mathrm{Ns}}, \mathrm{T} \times \mathrm{M}=0.302^{\mathrm{Ns}}$

Significance for FJR: $\mathrm{B}=0.020^{\mathrm{S}}, \mathrm{T}=0.640^{\mathrm{NS}}, \mathrm{M}=0.080^{\mathrm{NS}}, \mathrm{B} \times \mathrm{T}=0.187^{\mathrm{NS}}, \mathrm{B} \times \mathrm{M}=0.737^{\mathrm{NS}}, \mathrm{T} \times \mathrm{M}=0.461^{\mathrm{NS}}$

\begin{tabular}{|c|c|c|c|c|c|c|c|c|c|c|c|c|c|c|c|c|c|c|}
\hline \multicolumn{19}{|c|}{ Acidity $(\%)$} \\
\hline 0 & 0.98 & 0.98 & 0.98 & 0.97 & 0.98 & 0.97 & 0.99 & 0.98 & 0.98 & 0.98 & 0.98 & 0.99 & 0.98 & 0.98 & 0.98 & 0.99 & 0.97 & 0.99 \\
\hline 3 & 0.94 & 0.95 & 0.96 & 0.96 & 0.96 & 0.95 & 0.96 & 0.94 & 0.95 & 0.95 & 0.97 & 0.97 & 0.94 & 0.95 & 0.96 & 0.97 & 0.96 & 0.96 \\
\hline 6 & 0.92 & 0.91 & 0.93 & 0.94 & 0.95 & 0.93 & 0.94 & 0.90 & 0.92 & 0.93 & 0.95 & 0.94 & 0.92 & 0.91 & 0.93 & 0.94 & 0.94 & 0.94 \\
\hline Mean & 0.95 & 0.96 & 0.95 & 0.96 & 0.95 & 0.97 & 0.95 & 0.94 & 0.95 & 0.96 & 0.97 & 0.96 & 0.96 & 0.95 & 0.96 & 0.95 & 0.97 & 0.96 \\
\hline \multicolumn{19}{|c|}{$\begin{array}{l}\text { Significance for CHS: } \mathrm{B}=0.444^{\mathrm{NS}}, \mathrm{T}=0.265^{\mathrm{NS}}, \mathrm{M}=0.002^{\mathrm{s}}, \mathrm{B} \times \mathrm{T}=0.002^{\mathrm{s}}, \mathrm{B} \times \mathrm{M}=0.902^{\mathrm{NS}}, \mathrm{T} \times \mathrm{M}=0.005^{\mathrm{s}} \\
\text { Significance for FJR: } \mathrm{B}=0.129^{\mathrm{NS}}, \mathrm{T}=0.673^{\mathrm{Ns}}, \mathrm{M}=0.002^{\mathrm{s}}, \mathrm{B} \times \mathrm{T}=0.007^{\mathrm{s}}, \mathrm{B} \times \mathrm{M}=0.411^{\mathrm{NS}}, \mathrm{T} \times \mathrm{M}=0.120^{\mathrm{s}}\end{array}$} \\
\hline \multicolumn{19}{|c|}{ Non-enzymatic browning } \\
\hline 0 & 0.002 & 0.002 & 0.002 & 0.003 & 0.002 & 0.002 & 0.012 & 0.012 & 0.013 & 0.014 & 0.013 & 0.015 & 0.102 & 0.104 & 0.102 & 0.102 & 0.104 & 0.102 \\
\hline 3 & 0.032 & 0.030 & 0.031 & 0.034 & 0.034 & 0.029 & 0.043 & 0.041 & 0.044 & 0.047 & 0.046 & 0.047 & 0.121 & 0.119 & 0.124 & 0.127 & 0.121 & 0.121 \\
\hline 6 & 0.051 & 0.049 & 0.050 & 0.049 & 0.051 & 0.051 & 0.071 & 0.073 & 0.074 & 0.076 & 0.077 & 0.078 & 0.143 & 0.141 & 0.141 & 0.148 & 0.147 & 0.149 \\
\hline Mean & 0.028 & 0.027 & 0.028 & 0.029 & 0.029 & 0.028 & 0.042 & 0.042 & 0.044 & 0.046 & 0.045 & 0.047 & 0.122 & 0.121 & 0.122 & 0.126 & 0.124 & 0.124 \\
\hline \multicolumn{19}{|c|}{$\begin{array}{l}\text { Significance for CHS: } \mathrm{B}=0.407^{\mathrm{NS}}, \mathrm{T}=0.0001^{\mathrm{s}}, \mathrm{M}=0.001^{\mathrm{s}}, \mathrm{B} \times \mathrm{T}=0.628^{\mathrm{NS}}, \mathrm{B} \times \mathrm{M}=0.261^{\mathrm{NS}}, \mathrm{T} \times \mathrm{M}=0.0001^{\mathrm{s}} \\
\text { Significance for FJR: } \mathrm{B}=0.800^{\mathrm{Ns}}, \mathrm{T}=0.0001^{\mathrm{s}}, \mathrm{M}=0.001^{\mathrm{s}}, \mathrm{B} \times \mathrm{T}=0.358^{\mathrm{NS}}, \mathrm{B} \times \mathrm{M}=0.173^{\mathrm{NS}}, \mathrm{T} \times \mathrm{M}=0.0001^{\mathrm{s}}\end{array}$} \\
\hline \multicolumn{19}{|c|}{ L color value } \\
\hline 0 & 66.1 & 66.1 & 66.0 & 65.2 & 65.1 & 65.3 & 66.0 & 66.1 & 66.0 & 65.2 & 65.3 & 65.3 & 65.8 & 65.9 & 65.7 & 65.1 & 65.0 & 65.1 \\
\hline 3 & 65.8 & 65.7 & 65.8 & 65.1 & 65.0 & 65.1 & 65.8 & 65.9 & 65.8 & 65.1 & 65.2 & 65.1 & 65.1 & 65.5 & 65.4 & 64.7 & 64.7 & 64.6 \\
\hline 6 & 65.6 & 65.5 & 65.6 & 65.0 & 64.9 & 65.0 & 65.6 & 65.7 & 65.6 & 65.0 & 65.1 & 65.0 & 64.4 & 64.8 & 64.6 & 64.4 & 64.3 & 64.2 \\
\hline Mean & 65.8 & 65.8 & 65.6 & 65.1 & 65.0 & 65.1 & 65.8 & 65.8 & 65.8 & 65.1 & 65.2 & 65.1 & 65.1 & 65.4 & 65.2 & 64.7 & 64.7 & 64.6 \\
\hline
\end{tabular}

\begin{tabular}{llllllllllllllllllll}
\hline \multicolumn{11}{c}{ a color value } \\
\hline 0 & 26.7 & 26.7 & 26.6 & 27.4 & 27.3 & 27.4 & 26.7 & 26.7 & 26.8 & 27.4 & 27.3 & 27.4 & 26.3 & 26.4 & 26.3 & 26.7 & 27.3 & 26.7 \\
3 & 26.5 & 26.4 & 26.4 & 27.2 & 27.1 & 27.3 & 26.5 & 26.6 & 26.5 & 27.3 & 27.2 & 27.2 & 26.0 & 26.1 & 25.9 & 26.3 & 27.2 & 26.3 \\
6 & 26.4 & 26.3 & 26.2 & 27.1 & 27.0 & 27.2 & 26.4 & 26.5 & 26.4 & 27.2 & 27.1 & 27.0 & 25.9 & 25.8 & 25.7 & 25.9 & 27.1 & 25.8 \\
Mean & 26.5 & 26.5 & 26.4 & 27.2 & 27.1 & 27.3 & 26.5 & 26.6 & 26.6 & 27.3 & 27.1 & 27.2 & 26.1 & 26.1 & 26.0 & 26.3 & 27.2 & 26.3
\end{tabular}

Significance for CHS: $\mathrm{B}=0.101^{\mathrm{NS}}, \mathrm{T}=0.01^{\mathrm{s}}, \mathrm{M}=0.003^{\mathrm{s}}, \mathrm{B} \times \mathrm{T}=0.013^{\mathrm{s}}, \mathrm{B} \times \mathrm{M}=0.041^{\mathrm{s}}, \mathrm{T} \times \mathrm{M}=0.049^{\mathrm{s}}$

Significance for FJR: $\mathrm{B}=0.051^{\mathrm{NS}}, \mathrm{T}=0.003^{\mathrm{s}}, \mathrm{M}=0.038^{\mathrm{s}}, \mathrm{B} \times \mathrm{T}=0.000^{\mathrm{s}}, \mathrm{B} \times \mathrm{M}=0.039^{\mathrm{s}}, \mathrm{T} \times \mathrm{M}=0.031^{\mathrm{s}}$

\begin{tabular}{lllllllllllllllllll}
\hline \multicolumn{11}{c}{ b color value } \\
\hline 0 & 28.4 & 28.4 & 28.4 & 29.5 & 29.4 & 29.4 & 28.4 & 28.4 & 28.3 & 29.4 & 29.4 & 29.4 & 28.3 & 28.6 & 28.4 & 29.4 & 29.4 & 29.5 \\
3 & 28.2 & 28.1 & 28.2 & 29.3 & 29.2 & 29.2 & 28.2 & 28.1 & 28.1 & 29.1 & 29.2 & 29.1 & 28.1 & 28.2 & 28.1 & 29.0 & 29.2 & 29.1 \\
6 & 28.0 & 27.9 & 27.9 & 29.2 & 29.1 & 29.1 & 28.0 & 28.0 & 27.9 & 28.9 & 28.9 & 29.0 & 27.8 & 27.7 & 27.9 & 28.7 & 28.7 & 28.9 \\
Mean & 28.2 & 28.1 & 28.2 & 29.3 & 29.2 & 29.2 & 28.2 & 28.2 & 28.1 & 29.1 & 29.2 & 29.2 & 28.1 & 28.2 & 28.1 & 29.0 & 29.1 & 29.2
\end{tabular}

Significance for CHS: B $=0.805^{\mathrm{Ns}}, \mathrm{T}=0.043^{\mathrm{s}}, \mathrm{M}=0.004^{\mathrm{s}}, \mathrm{B} \times \mathrm{T}=0.040^{\mathrm{s}}, \mathrm{B} \times \mathrm{M}=0.049^{\mathrm{s}}, \mathrm{T} \times \mathrm{M}=0.047^{\mathrm{s}}$

Significance for FJR: $\mathrm{B}=0.790^{\mathrm{NS}}, \mathrm{T}=0.048^{\mathrm{s}}, \mathrm{M}=0.005^{\mathrm{s}}, \mathrm{B} \times \mathrm{T}=0.012^{\mathrm{s}}, \mathrm{B} \times \mathrm{M}=0.048^{\mathrm{s}}, \mathrm{T}$ x M $=0.001^{\mathrm{s}}$

CHS: Chaunsa cv., FJR: Fajri cv., OD: Osmatic dehydration, IR: Infrared blanching, MW: Microwave blanching, B: Blanching, T: Treatment, M: Month, NS: Not significant, S: Significant 
TABLE 2. Effect of treatments and storage on the nutritional value of dried mango slices.

\begin{tabular}{|c|c|c|c|c|c|c|c|c|c|c|c|c|c|c|c|c|c|c|}
\hline \multirow{3}{*}{$\begin{array}{l}\text { Storage } \\
\text { period } \\
\text { (months) }\end{array}$} & \multicolumn{6}{|c|}{ Potassium metabisulphite $6 \%$ (KMS) } & \multicolumn{6}{|c|}{ Potassium sorbates 350 ppm (PS) } & \multicolumn{6}{|c|}{ Control } \\
\hline & \multicolumn{3}{|c|}{ CHS } & \multicolumn{3}{|c|}{ FJR } & \multicolumn{3}{|c|}{ CHS } & \multicolumn{3}{|c|}{ FJR } & \multicolumn{3}{|c|}{ CHS } & \multicolumn{3}{|c|}{ FJR } \\
\hline & OD & IR & MW & OD & IR & MW & OD & IR & MW & OD & IR & MW & OD & IR & MW & OD & IR & MW \\
\hline \multicolumn{19}{|c|}{ Protein $(\%)$} \\
\hline 0 & 1.06 & 1.04 & 1.05 & 1.08 & 1.07 & 1.08 & 1.08 & 1.07 & 1.06 & 1.08 & 1.07 & 1.0 & 1.08 & 1.06 & 1.06 & 1.08 & 1.08 & 1.08 \\
\hline 3 & 1.08 & 1.07 & 1.07 & 1.09 & 1.08 & 1.08 & 1.08 & 1.08 & 1.08 & 1.09 & 1.08 & 1.08 & 1.07 & 1.07 & 1.07 & 1.07 & 1.08 & 1.08 \\
\hline 6 & 1.06 & 1.09 & 1.08 & 1.07 & 1.08 & 1.08 & 1.07 & 1.07 & 1.07 & 1.07 & 1.08 & 1.08 & 1.09 & 1.05 & 1.06 & 1.08 & 1.09 & 1.09 \\
\hline Mean & 1.07 & 1.07 & 1.07 & 1.08 & 1.08 & 1.08 & 1.08 & 1.07 & 1.07 & 1.08 & 1.08 & 1.08 & 1.08 & 1.06 & 1.06 & 1.08 & 1.08 & 1.08 \\
\hline
\end{tabular}

Significance for CHS: $\mathrm{B}=0.156^{\mathrm{NS}}, \mathrm{T}=0.593^{\mathrm{NS}}, \mathrm{M}=0.353^{\mathrm{NS}}, \mathrm{B} \times \mathrm{T}=0.596^{\mathrm{NS}}, \mathrm{B} \times \mathrm{M}=0.776^{\mathrm{Ns}}, \mathrm{T} \times \mathrm{M}=0.307^{\mathrm{NS}}$

Significance for FJR: $\mathrm{B}=0.819^{\mathrm{NS}}, \mathrm{T}=0.819^{\mathrm{NS}}, \mathrm{M}=0.819^{\mathrm{NS}}, \mathrm{B} \times \mathrm{T}=0.232^{\mathrm{NS}}, \mathrm{B} \times \mathrm{M}=0.029^{\mathrm{s}}, \mathrm{T} \times \mathrm{M}=0.029^{\mathrm{s}}$

\begin{tabular}{lllllllllllllllllll}
\multicolumn{11}{c}{ Ash $(\%)$} \\
\hline 0 & 3.3 & 3.2 & 3.3 & 3.9 & 3.8 & 3.8 & 3.3 & 3.2 & 3.3 & 3.9 & 3.8 & 3.8 & 3.3 & 3.2 & 3.3 & 3.9 & 3.8 & 3.8 \\
3 & 3.2 & 3.3 & 3.2 & 3.9 & 3.9 & 3.9 & 3.3 & 3.3 & 3.4 & 3.8 & 3.8 & 3.9 & 3.3 & 3.3 & 3.2 & 3.9 & 3.9 & 3.9 \\
6 & 3.3 & 3.3 & 3.3 & 3.8 & 3.9 & 3.9 & 3.3 & 3.3 & 3.3 & 3.8 & 3.9 & 3.9 & 3.3 & 3.3 & 3.3 & 3.8 & 3.9 & 3.9 \\
Mean & 3.3 & 3.3 & 3.3 & 3.9 & 3.9 & 3.9 & 3.3 & 3.3 & 3.3 & 3.8 & 3.8 & 3.9 & 3.3 & 3.3 & 3.3 & 3.9 & 3.9 & 3.9
\end{tabular}

Significance for CHS: $\mathrm{B}=0.751 \mathrm{NS}, \mathrm{T}=1.000 \mathrm{NS}, \mathrm{M}=0.781 \mathrm{NS}, \mathrm{B} \times \mathrm{T}=1.000 \mathrm{NS}, \mathrm{B} \times \mathrm{M}=0.865 \mathrm{NS}, \mathrm{T} \times \mathrm{M}=0.244 \mathrm{NS}$

Significance for FJR: B $=0.965 \mathrm{NS}, \mathrm{T}=0.444 \mathrm{NS}, \mathrm{M}=0.679 \mathrm{NS}, \mathrm{B} \times \mathrm{T}=0.461 \mathrm{NS}, \mathrm{B} \times \mathrm{M}=0.0003 \mathrm{NS}, \mathrm{T} \times \mathrm{M}=0.045 \mathrm{NS}$

\begin{tabular}{|c|c|c|c|c|c|c|c|c|c|c|c|c|c|c|c|c|c|c|}
\hline \multicolumn{19}{|c|}{ Fiber $(\%)$} \\
\hline 0 & 1.9 & 1.8 & 1.9 & 1.8 & 1.9 & 1.9 & 1.8 & 1.9 & 1.8 & 1.9 & 1.9 & 1.9 & 1.9 & 1.9 & 1.9 & 1.9 & 1.9 & 1.9 \\
\hline 3 & 1.9 & 1.9 & 1.8 & 1.9 & 1.9 & 1.9 & 1.9 & 1.8 & 1.9 & 1.8 & 1.9 & 1.9 & 1.9 & 1.9 & 1.9 & 1.9 & 1.9 & 1.9 \\
\hline 6 & 1.8 & 1.9 & 1.9 & 1.9 & 1.8 & 1.8 & 1.9 & 1.9 & 1.9 & 1.9 & 1.8 & 1.8 & 1.8 & 1.8 & 1.8 & 1.8 & 1.8 & 1.8 \\
\hline Mean & 1.9 & 1.9 & 1.9 & 1.9 & 1.9 & 1.9 & 1.9 & 1.9 & 1.9 & 1.9 & 1.9 & 1.9 & 1.9 & 1.9 & 1.9 & 1.9 & 1.9 & 1.9 \\
\hline
\end{tabular}

Significance for CHS: $\mathrm{B}=1.000 \mathrm{NS}, \mathrm{T}=0.444 \mathrm{NS}, \mathrm{M}=0.670 \mathrm{NS}, \mathrm{B} \times \mathrm{T}=0.461 \mathrm{NS}, \mathrm{B} \times \mathrm{M}=0.073 \mathrm{NS}, \mathrm{T} \times \mathrm{M}=0.232 \mathrm{NS}$

Significance for FJR: $\mathrm{B}=1.000 \mathrm{NS}, \mathrm{T}=0.444 \mathrm{NS}, \mathrm{M}=0.670 \mathrm{NS}, \mathrm{B} \times \mathrm{T}=0.461 \mathrm{NS}, \mathrm{B} \times \mathrm{M}=0.073 \mathrm{NS}, \mathrm{T} \times \mathrm{M}=0.232 \mathrm{NS}$

\begin{tabular}{|c|c|c|c|c|c|c|c|c|c|c|c|c|c|c|c|c|c|c|}
\hline \multicolumn{19}{|c|}{ Reducing sugars (\%) } \\
\hline 0 & 2.9 & 2.8 & 2.9 & 3.1 & 3.1 & 3.0 & 3.2 & 3.3 & 3.3 & 3.5 & 3.6 & 3.5 & 3.4 & 3.3 & 3.2 & 3.7 & 3.6 & 3.5 \\
\hline 3 & 3.7 & 3.5 & 3.6 & 4.2 & 4.2 & 4.3 & 3.9 & 4.1 & 4.2 & 4.6 & 4.7 & 4.6 & 6.1 & 6.1 & 6.1 & 4.8 & 4.7 & 4.8 \\
\hline 6 & 3.9 & 3.8 & 3.8 & 4.5 & 4.5 & 4.5 & 4.1 & 4.4 & 4.3 & 4.8 & 4.7 & 4.4 & 6.7 & 6.7 & 6.6 & 5.1 & 5.1 & 5.1 \\
\hline Mean & 3.5 & 3.4 & 3.4 & 3.9 & 3.9 & 3.9 & 3.7 & 3.9 & 3.9 & 4.3 & 4.3 & 4.2 & 5.4 & 5.4 & 5.3 & 4.5 & 4.5 & 4.5 \\
\hline \multicolumn{19}{|c|}{$\begin{array}{l}\text { Significance for CHS: } \mathrm{B}=0.927^{\mathrm{NS}}, \mathrm{T}=0.071^{\mathrm{NS}}, \mathrm{M}=0.085^{\mathrm{NS}}, \mathrm{B} \times \mathrm{T}=0.001^{\mathrm{s}}, \mathrm{B} \times \mathrm{M}=0.175^{\mathrm{NS}}, \mathrm{T} \times \mathrm{M}=0.0001^{\mathrm{s}} \\
\text { Significance for FJR: } \mathrm{B}=0.303^{\mathrm{NS}}, \mathrm{T}=0.010^{\mathrm{s}}, \mathrm{M}=0.0001^{\mathrm{s}}, \mathrm{B} \times \mathrm{T}=0.397^{\mathrm{NS}}, \mathrm{B} \times \mathrm{M}=0.461^{\mathrm{NS}}, \mathrm{T} \times \mathrm{M}=0.019^{\mathrm{s}}\end{array}$} \\
\hline \multicolumn{19}{|c|}{ Non-reducing sugars (\%) } \\
\hline 0 & 10.8 & 10.8 & 10.7 & 9.8 & 9.7 & 9.8 & 10.4 & 10.2 & 10.3 & 9.4 & 9.2 & 9.1 & 10.2 & 10.1 & 10.1 & 9.1 & 9.1 & 9.0 \\
\hline 3 & 9.9 & 9.8 & 9.8 & 8.6 & 8.4 & 8.4 & 9.2 & 8.9 & 8.8 & 8.1 & 8.0 & 8.9 & 7.1 & 7.1 & 7.1 & 7.8 & 7.7 & 7.4 \\
\hline 6 & 9.6 & 9.4 & 9.5 & 8.1 & 8.0 & 8.1 & 8.9 & 8.4 & 8.5 & 7.8 & 7.8 & 7.7 & 6.3 & 6.2 & 6.2 & 7.2 & 7.1 & 7.2 \\
\hline Mean & 10.1 & 10.0 & 10.0 & 8.8 & 8.8 & 8.8 & 9.5 & 9.2 & 9.2 & 8.4 & 8.3 & 8.6 & 7.9 & 7.8 & 7.8 & 8.03 & 8.0 & 7.9 \\
\hline $\begin{array}{l}\text { Signific } \\
\text { Signific }\end{array}$ & $\begin{array}{l}\mathrm{CH} \\
\mathrm{FJR}\end{array}$ & $\begin{array}{l}\mathrm{B}= \\
\mathrm{B}=0\end{array}$ & $\begin{array}{ll}020^{\mathrm{s}}, \\
273^{\mathrm{NS}},\end{array}$ & $=0$. & NS, N & 0.0 & $2^{\mathrm{s}}, \mathrm{B} x$ & $=0.0$ & $2^{\mathrm{s}}, \mathrm{B}$ & $I=$ & $309^{\mathrm{NS}}$ & $\mathrm{xN}$ & 0.00 & & & & & \\
\hline
\end{tabular}

observed while the treatment had a significant effect on color values during the storage as compared to the untreated mango dried slices. It is evidenced that KMS and PS have a beneficial effect on the color of dried mango slices due to their anti-browning effect in foods [Sra et al., 2014]. The L, $\mathrm{a}$ and $\mathrm{b}$ color values of dried mango slices were significantly $(p<0.05)$ influenced during storage for both the cultivars. A significant decrease $(p<0.05)$ in $\mathrm{L}$, a and b color values was noticed up to 3 months of storage (Table 1) while slight reduction was observed in the last three months. Drying did not influence the color of dried mango slices subject to different blanching techniques (IR and MW) and OD prior to hot air drying. Prevention of color changes during storage could be associated with inhibition of PPO activity in OD samples or inactivation of PPO in blanched samples, since PPO enzyme is responsible for the browning of dried foods [Ioannou
\& Ghoul, 2013; Yadav \& Singh, 2014]. Several reactions such as pigment degradation especially of carotenoids, oxidation of ascorbic acid and Maillard reaction occur during thermal treatments. Oxidation of ascorbic acid and Maillard reaction produce yellow brown color in dried mango slices [Chong et al., 2013; Korbel et al., 2013] while this change in color is absent in OD and blanched dried mango slices. The mean value of non-enzymatic browning recorded for KMS-treated dried mango slices (both cultivars) after 6-month storage was lower as compared to the PS-treated and control (untreated) samples. A significant difference $(p<0.05)$ was found for non-enzymatic browning between treated and untreated (control) dried mango slices. The lower value of non-enzymatic browning for KMS-treated dried mango slices might be due to the antioxidant effect of KMS. Sulphites have been reported as non-enzymatic 
TABLE 2. continued

\begin{tabular}{|c|c|c|c|c|c|c|c|c|c|c|c|c|c|c|c|c|c|c|}
\hline \multirow{3}{*}{$\begin{array}{l}\text { Storage } \\
\text { period } \\
\text { (months) }\end{array}$} & \multicolumn{6}{|c|}{ Potassium metabisulphite $6 \%$ (KMS) } & \multicolumn{6}{|c|}{ Potassium sorbates $350 \mathrm{ppm}(\mathrm{PS})$} & \multicolumn{6}{|c|}{ Control } \\
\hline & \multicolumn{3}{|c|}{$\mathrm{CHS}$} & \multicolumn{3}{|c|}{ FJR } & \multicolumn{3}{|c|}{ CHS } & \multicolumn{3}{|c|}{ FJR } & \multicolumn{3}{|c|}{$\mathrm{CHS}$} & \multicolumn{3}{|c|}{ FJR } \\
\hline & OD & IR & MW & OD & IR & MW & OD & IR & MW & OD & IR & MW & OD & IR & MW & $\mathrm{OD}$ & $\mathrm{IR}$ & MW \\
\hline \multicolumn{19}{|c|}{ Total sugars $(\%)$} \\
\hline 0 & 13.7 & 13.6 & 13.6 & 12.9 & 12.8 & 12.8 & 13.6 & 13.5 & 13.6 & 12.8 & 12.8 & 12.6 & 13.6 & 13.4 & 13.4 & 12.8 & 12.7 & 12.5 \\
\hline 3 & 13.6 & 13.3 & 13.4 & 12.8 & 12.6 & 12.7 & 13.1 & 13.0 & 13.0 & 12.7 & 12.7 & 12.5 & 13.2 & 13.2 & 13.2 & 12.6 & 12.4 & 12.4 \\
\hline 6 & 13.5 & 13.2 & 13.3 & 12.6 & 12.5 & 12.6 & 13.0 & 12.8 & 12.8 & 12.6 & 12.5 & 12.1 & 13.0 & 12.9 & 12.8 & 12.3 & 12.2 & 12.3 \\
\hline Mean & 13.6 & 13.4 & 13.4 & 12.8 & 12.6 & 12.7 & 13.2 & 13.1 & 13.1 & 12.7 & 12.7 & 12.4 & 13.3 & 13.2 & 13.1 & 12.6 & 12.4 & 12.4 \\
\hline \multicolumn{19}{|c|}{$\begin{array}{l}\text { Significance for CHS: } \mathrm{B}=0.006^{\mathrm{S}}, \mathrm{T}=0.058^{\mathrm{NS}}, \mathrm{M}=0.016^{\mathrm{S}}, \mathrm{B} \times \mathrm{T}=0.431^{\mathrm{NS}}, \mathrm{B} \times \mathrm{M}=0.642^{\mathrm{NS}}, \mathrm{T} \times \mathrm{M}=0.003^{\mathrm{S}} \\
\text { Significance for FJR: } \mathrm{B}=0.003^{\mathrm{s}}, \mathrm{T}=0.005^{\mathrm{s}}, \mathrm{M}=0.0001^{\mathrm{s}}, \mathrm{B} \times \mathrm{T}=0.091^{\mathrm{NS}}, \mathrm{B} \times \mathrm{M}=0.896^{\mathrm{NS}}, \mathrm{T} \times \mathrm{M}=0.666^{\mathrm{NS}}\end{array}$} \\
\hline \multicolumn{19}{|c|}{ Ascorbic acid $(\mathrm{mg} / 100 \mathrm{~g})$} \\
\hline 0 & 127.1 & 88.2 & 99.3 & 87.4 & 52.7 & 61.6 & 126.3 & 86.1 & 95.2 & 84.2 & 49.7 & 58.3 & 118.1 & 77.1 & 87.2 & 78.2 & 41.7 & 51.3 \\
\hline 3 & 122.5 & 84.7 & 97.4 & 86.2 & 51.2 & 59.9 & 120.4 & 83.2 & 91.3 & 79.4 & 44.7 & 53.2 & 106.4 & 71.2 & 81.1 & 71.5 & 39.6 & 48.2 \\
\hline 6 & 120.6 & 82.5 & 96.3 & 85.3 & 49.8 & 58.3 & 117.7 & 79.3 & 89.4 & 76.4 & 41.3 & 51.1 & 101.7 & 68.4 & 78.4 & 67.4 & 36.3 & 43.6 \\
\hline Mean & 123.4 & 85.1 & 97.7 & 86.3 & 51.2 & 59.9 & 121.5 & 82.9 & 92.0 & 80.0 & 45.2 & 54.2 & 108.7 & 72.2 & 82.2 & 72.4 & 39.2 & 47.7 \\
\hline \multicolumn{19}{|c|}{$\begin{array}{l}\text { Significance for CHS: } \mathrm{B}=0.0001^{\mathrm{s}}, \mathrm{T}=0.001^{\mathrm{s}}, \mathrm{M}=0.008^{\mathrm{s}}, \mathrm{B} \times \mathrm{T}=0.044^{\mathrm{s}}, \mathrm{B} \times \mathrm{M}=0.029^{\mathrm{s}}, \mathrm{T} \times \mathrm{M}=0.007^{\mathrm{s}} \\
\text { Significance for FJR: } \mathrm{B}=0.0001^{\mathrm{s}}, \mathrm{T}=0.001^{\mathrm{s}}, \mathrm{M}=0.025^{\mathrm{s}}, \mathrm{B} \times \mathrm{T}=0.502^{\mathrm{Ns}}, \mathrm{B} \times \mathrm{M}=0.761^{\mathrm{Ns}}, \mathrm{T} \times \mathrm{M}=0.011^{\mathrm{s}}\end{array}$} \\
\hline \multicolumn{19}{|c|}{ Total carotenoids (mg/100 g) } \\
\hline 0 & 7.8 & 7.7 & 7.8 & 5.2 & 5.1 & 5.0 & 7.5 & 7.4 & 7.5 & 4.9 & 4.9 & 4.8 & 7.4 & 7.2 & 7.4 & 4.8 & 4.7 & 4.7 \\
\hline 3 & 6.6 & 6.5 & 6.4 & 4.6 & 4.5 & 4.5 & 6.3 & 6.3 & 6.2 & 4.3 & 4.4 & 4.3 & 6.2 & 6.1 & 6.0 & 4.2 & 4.3 & 4.2 \\
\hline 6 & 6.3 & 6.3 & 6.2 & 4.2 & 4.3 & 4.2 & 5.9 & 5.9 & 5.8 & 3.9 & 4.0 & 3.9 & 5.8 & 5.7 & 5.7 & 3.8 & 3.9 & 3.8 \\
\hline Mean & 6.9 & 6.8 & 6.8 & 4.7 & 4.6 & 4.6 & 6.6 & 6.5 & 6.5 & 4.4 & 4.4 & 4.3 & 6.5 & 6.3 & 6.4 & 4.3 & 4.3 & 4.2 \\
\hline
\end{tabular}

CHS: Chaunsa cv., FJR: Fajri cv., OD: Osmatic dehydration, IR: Infrared blanching, MW: Microwave blanching, B: Blanching, T: Treatment, M: Month, NS: Not significant, S: Significant

and enzymatic browning inhibitors. A significant effect $(p<0.05)$ of storage was also observed on non-enzymatic browning while no significant difference was found among the blanching techniques for both the cultivars. The moisture content in both the cultivars of dried mango slices was increasing with time and this increase might be due to the higher permeability of HDPE bags to moisture [Sra et al., 2014]. The increment in moisture content was significant $(p<0.05)$ for CHS cultivar, while no significant $(p<0.05)$ effect was observed in the case of FJR cultivar. The moisture content increment in CHS cultivar was not significantly affected by the blanching technique but a significant effect was observed for FJR cultivar (Table 1). The mean value of ash content was found to range between $3.2-3.3 \%$ and $3.8-3.9 \%$ (Table 2) for CHS and FJR cultivar, respectively, while the mean values of fiber and protein content were $1.9 \%$ and $1.06-1.08 \%$ (Table 2), respectively, for both the cultivars. No significant difference was observed in ash, fiber and protein contents as affected by storage period, blanching technique and treatment. Significant decline $(p<0.05)$ in acidity was noticed during the storage (Table 1). The decrease in acidity might be due to the biochemical interaction in bind-

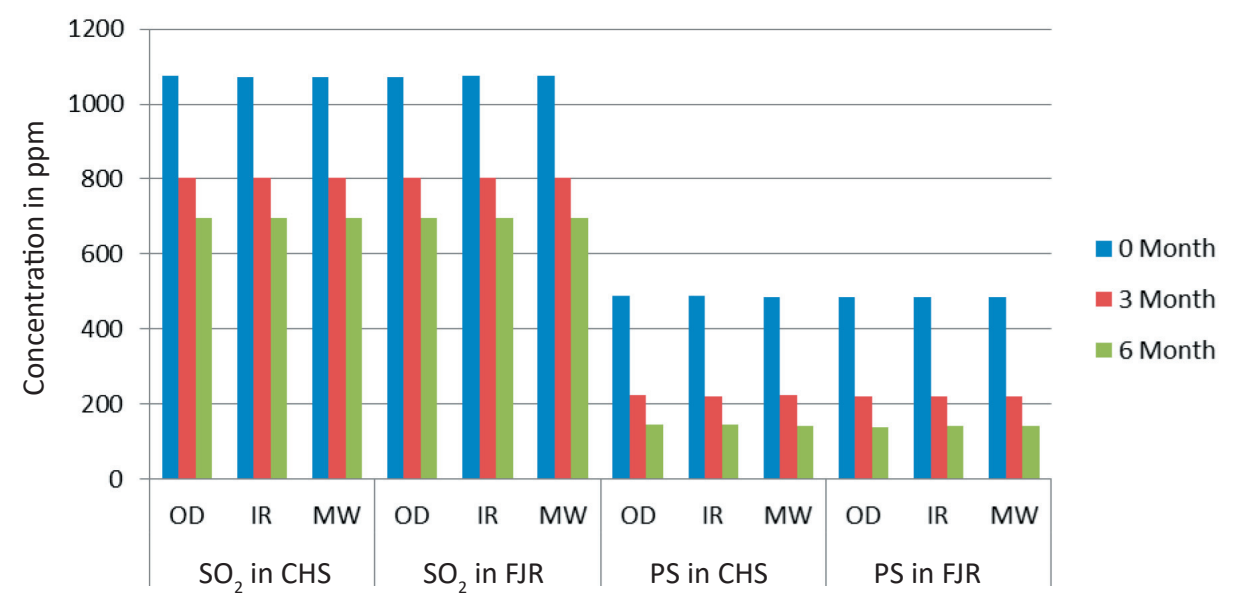

FIGURE 1. Effect of treatments and storage on $\mathrm{SO}_{2}$ and potassium sorbate retention in dried mango slices. CHS: Chaunsa cv., FJR: Fajri cv., OD: Osmatic dehydration, IR: Infrared blanching, MW: Microwave blanching. 

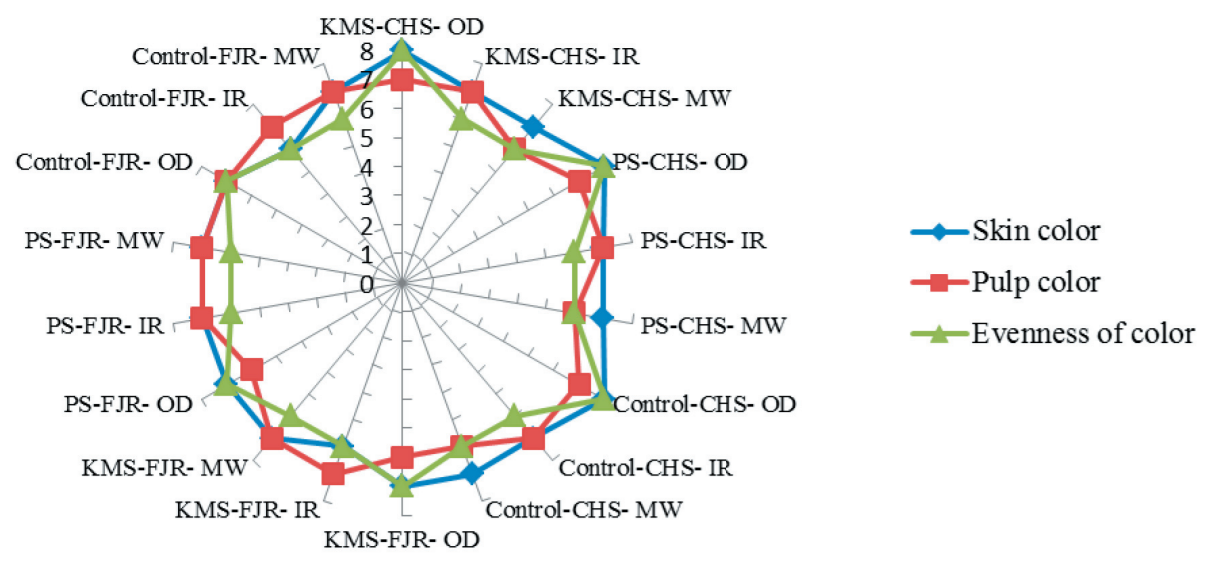

FIGURE 2. Descriptive sensory analysis of appearance attributes of dried mango slices as affected by blanching and treatments. CHS: Chaunsa cv., FJR: Fajri cv., OD: Osmatic dehydration, IR: Infrared blanching, MW: Microwave blanching.

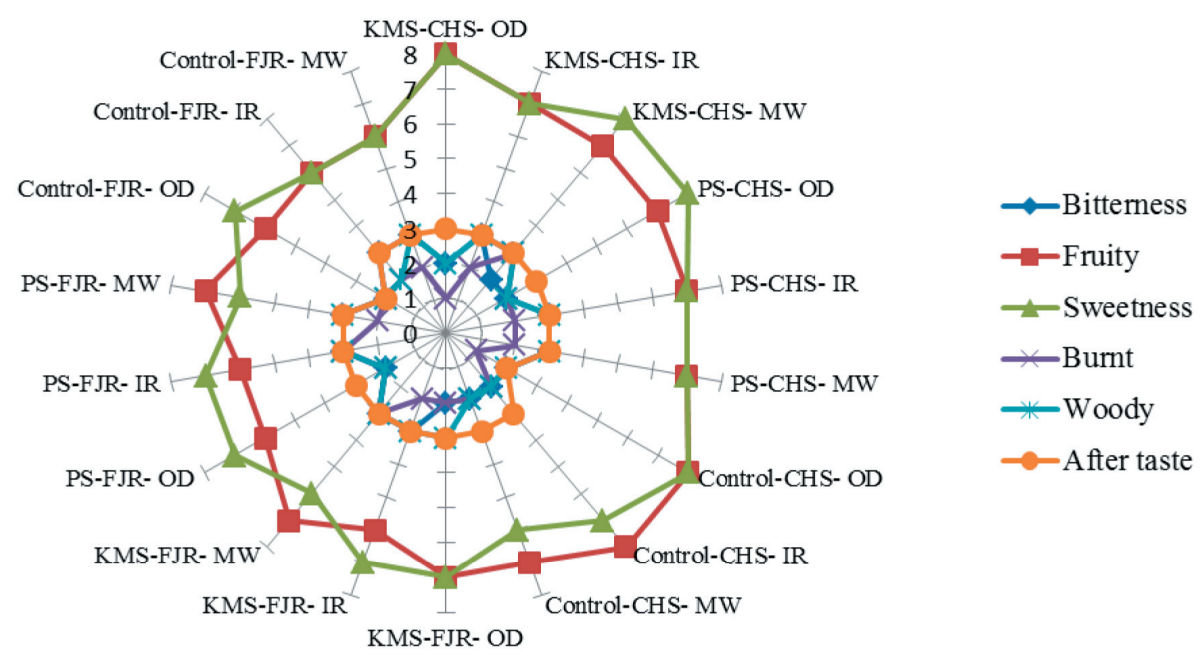

FIGURE 3. Descriptive sensory analysis of flavor attributes of dried mango slices as affected by blanching and treatments. CHS: Chaunsa cv., FJR: Fajri cv., OD: Osmatic dehydration, IR: Infrared blanching, MW:Microwave blanching.

ing of acid with other components with passage of time. Acid content of mango powder progressively decreases during storage even at room temperature $\left(25 \pm 5^{\circ} \mathrm{C}\right)$ as reported earlier [Aktas et al., 2013]. CHS cultivar has higher sugar contents than the FJR cultivar and significant differences in sugar contents (reducing sugar, non-reducing sugar, total sugar) were noticed for treatments, storage, blanching technique and among the interaction of these variables. Mean content of reducing sugars increased significantly $(p<0.05)$ for both the cultivars during storage. The reducing sugar increment in treated dried mango slices is attributable to slow inversion of starch and non-reducing sugars into reducing sugars. The increase in reducing sugar and a decrease in non-reducing sugar was significantly affected $(p<0.05)$ by treatments (KMS, PS and untreated). Total sugar retention in KMStreated dried mango slices was significantly higher than in the PS-treated ones. It is evident that KMS helped in lowering the inversion of non-reducing sugars to reducing sugars and had a beneficial effect on the retention of total sugars. Total sugars reduction in dried mango slices indicates the possibility of their browning and biochemical reactions
[Sra et al., 2014]. The contents of ascorbic acid (vitamin C) for both cultivars after OD, IR and MW blanching are presented in Table 2. The vitamin $\mathrm{C}$ retention in OD mango slices was higher than in the IR and MW blanched dried mango slices but the retention of vitamin $\mathrm{C}$ content of both cultivars was observed to be lower than in the fresh mango samples (CHS: $135 \mathrm{mg} / 100 \mathrm{~g}$, FJR: $94 \mathrm{mg} / 100 \mathrm{~g}$ ). This loss was attributed to leaching and chemical degradation of vitamin $\mathrm{C}$ to the osmotic solution and drying process [ArayaFarias et al., 2014; Yadav \& Singh, 2014]. The treatment of dried mango slices had a significant $(p<0.05)$ effect on the retention of vitamin $\mathrm{C}$. The KMS- and PS-treated dried mango slices had higher retention of vitamin $\mathrm{C}$ as compared to the untreated dried mango slices. The higher retention of vitamin $\mathrm{C}$ in the KMS-treated dried mango slices was attributed to its antioxidative potential as compared to the PS which prevents the conversion of ascorbic acid to dehydroascorbic acid. Retention of vitamin C in MW blanched dried mango slices was significantly higher $(p<0.05)$ than in the IR blanched dried mango slices and these results are in agreement with previous findings made for vegetables blanched 


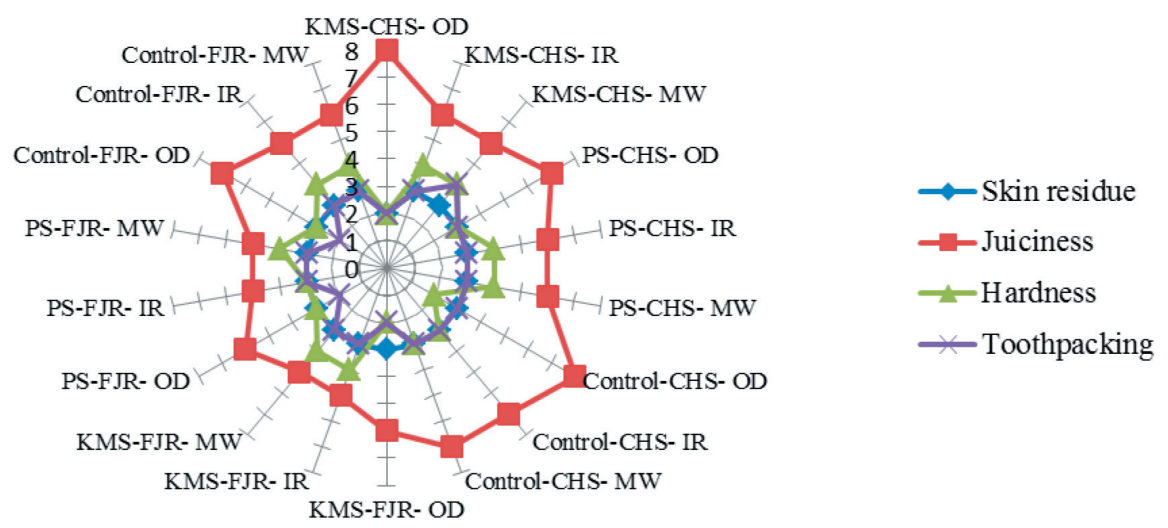

FIGURE 4. Descriptive sensory analysis of texture attributes of dried mango slices as affected by blanching and treatments. CHS: Chaunsa cv., FJR: Fajri cv., OD: Osmatic dehydration, IR: Infrared blanching, MW: Microwave blanching.

by both the techniques [Jeevitha et al., 2015; Ruiz-Ojeda \& Peñas, 2013; Vishwanathan et al., 2013]. The total content of $\beta$-carotenoids is the sum of all trans and cis carotenoids and not much studies are still available in literature for their stability at IR and MW blanching. A significant loss $(p<0.05)$ was noticed in $\beta$-carotenoids contents of both the cultivars with passage of time. Carotenoids are susceptible to oxidative loss caused by dry heat which is responsible for this loss during storage. Treatments of dried mango slices had a significant $(p<0.05)$ effect on the retention of carotenoids. Carotenoids destruction rate reduced in sulphite-treated dried mango slices due to the protective effect of $\mathrm{SO}_{2}$. Total carotenoid content in OD mango slices was bit higher than in the IR and MW blanched dry mango slices, which may be associated with its hydrophobic characteristics. Carotenoids are prone to isomerization due to their highly unsaturated structure and this phenomenon becomes more pronounced during processing and storage [Qian et al., 2012]. The retention of $\mathrm{SO}_{2}$ was significantly affected $(p<0.05)$ during storage of dried mango slices, a substantial amount of $\mathrm{SO}_{2}$ was lost over the 6-month storage period (Figure 1) and this loss in $\mathrm{SO}_{2}$ was also due to its leakage from HDPE bags. The loss of $\mathrm{SO}_{2}$ was up to $25.0 \%$ in the first three months in both the cultivars and in the last three months it was up to $13.5 \%$. PS concentration was also significantly decreased $(p<0.05)$. It was lost up to 4 fold at 6 months (Figure 1) of storage time and this loss is attributed to the oxidation of PS. The permeable nature of HDPE accelerates the PS oxidation [Sra et al., 2014].

\section{Sensory evaluation}

In total, 13 sensory attributes were evaluated for dried mango slices of both cultivars after the storage period of 6 months. The organoleptic scores did not vary significantly with treatments and blanching techniques. It is evident from Figure 2 that the appearance of dried mango slices after 6-month storage did not show any significant differences among the color properties viz. skin color, pulp color and evenness of color. The OD samples received 7-8 score while the IR and MW blanched samples received 6-7 score for all appearance attributes. Figure 3 describes the flavor profile of dried mango slices, again the OD samples achieved the highest score for sweetness and juiciness (7-8). In the IR and MW blanched samples, burnt (2), bitterness (3), woody (3), after taste (3) flavors were noticed regardless the treatment. The dried mango slices of both cultivars had the same intensity of texture attributes (Figure 4). The IR and MW blanched samples had a higher tooth packing intensity (3-4) as compared to the OD samples (2). Juiciness prevailed in the OD samples (8), while skin residue and hardness scores were higher in the IR and MW blanched samples than in the OD samples of both cultivars. From the sensory point of view it was concluded that both the cultivars of mango are suitable for preparation of dry slices.

\section{Microbiological analysis}

Slight variation in microbial counts was noticed in KMS, PS and untreated dried mango slices. A very low count was detected, which might be due to the preservative effect of $\mathrm{SO}_{2}$

TABLE 3. Microbiological analyses of dried mango slices.

\begin{tabular}{|c|c|c|c|c|c|c|c|c|c|c|c|c|}
\hline \multirow{3}{*}{ Treatment } & \multicolumn{6}{|c|}{ Yeast (cfu/g) } & \multicolumn{6}{|c|}{ Mold (cfu/g) } \\
\hline & \multicolumn{3}{|c|}{ CHS } & \multicolumn{3}{|c|}{ FJR } & \multicolumn{3}{|c|}{ CHS } & \multicolumn{3}{|c|}{ FJR } \\
\hline & OD & IR & MW & OD & IR & MW & OD & IR & MW & OD & IR & MW \\
\hline Potassium metabisulphite $6 \%$ (KMS) & 8 & 4 & 3 & 2 & 1 & 1 & - & - & - & - & - & - \\
\hline Potassium sorbates $350 \mathrm{ppm}$ (PS) & - & - & - & - & - & - & - & - & - & - & - & - \\
\hline Control & 12 & 10 & 9 & 11 & 9 & 11 & 25 & 16 & 15 & 13 & 12 & 11 \\
\hline
\end{tabular}

CHS: Chaunsa cv., FJR: Fajri cv., OD: Osmatic dehydration, IR: Infrared blanching, MW: Microwave blanching 
and PS. Also the $\mathrm{a}_{\mathrm{w}}$ ranged between 0.63 and 0.71 during 6-month storage at which the growth of organisms was not possible. In addition, in the range of 9-12 and 11-25 cfu/g yeast and mold count was observed in the untreated dried mango slices respectively, while $1-8 \mathrm{cfu} / \mathrm{g}$ yeast was detected in KMS-treated dried mango slices and no growth of yeast and mould was detected in PS-treated dried mango slices (Table 3). The yeast and mold counts detected in present study for dried mango slices were below the permissible limit set by the International Commission for Microbial Specifications for Foods [Sra et al., 2014].

\section{CONCLUSIONS}

It was concluded that under the ambient conditions the dried mango slices can be stored for the period of 6 months. Between the treatments applied in the study, i.e. IR and MW blanching followed by immersion in KMS and PS as well as OD, the $6 \%$ KMS treated samples resulted in better preservation of physiochemical characteristics during storage. From the microbiological point of view, all the dried mango slices of the cultivars were safe. On the other hand, the OD samples had better retention of $\beta$-carotenoids and vitamin $C$ while MW blanched samples showed higher retention of vitamin $C$ than IR blanched samples. Consequently, both the cultivars of mango have excellent nutritional qualities and consumer will enjoy eating these dried mango slices because of their adequate sensory quality.

\section{RESEARCH FUNDING}

There has been no financial support for this work that could have influenced its outcome.

\section{CONFLICT OF INTERESTS}

Authors declare no conflict of interests.

\section{REFERENCES}

1. Ahmad S.A., Ahmed M., Qadir M.A., Shafiq M.I., Batool N., Nosheen N., Ahmad M., Mahmood R.K., Khokhar Z.U., Quantitation and Risk Assessment of Chemical Adulterants in Milk Using UHPLC Coupled to Photodiode Array and Differential Refractive Index Detectors. Food Anal. Methods, 2016, 9, 3367-3376.

2. Ahmed M., Khaleeq A., Huma R., Qadir M.A., Shafiq M.I., Israr A., Ali A., Shahzad S., Optimization and Validation Procedure for Elemental Composition of Fresh and Pasteurized Milk in Pakistan Employing Microwave Digestion Followed by ICPOES: a Contribution to Risk Assessment. Food Anal. Methods, 2016a, 9, 2933-2942.

3. Ahmed M., Shafiq M.I., Khaleeq A., Huma R., Abdul Qadir M., Khalid A., Ali A., Samad A., Physiochemical, Biochemical, Minerals Content Analysis, and Antioxidant Potential of National and International Honeys in Pakistan. J. Chem., 2016b, art. no. 8072305.

4. Ahmed M., Shahzadi S.K., Waseem R., Shahzad S., Ahmad W., Sodium Benzoate and Potassium Sorbate in Pakistani Retail Foodstuffs. Intern. J. Chem. Sci. Res., 2013, 3, 1-6.
5. Aktas T., Ulger P., Daglioglu F., Hasturk F., Changes of Nutritional and Physical Quality Characteristics during Storage of Osmotic Pretreated Apple before Hot Air Drying and Sensory Evaluation. J. Food Quality, 2013, 36, 411-425.

6. Araya-Farias M., Macaigne O., Ratti C., On the development of osmotically dehydrated seabuckthorn fruits: Pretreatments, osmotic dehydration, postdrying techniques, and nutritional quality. Drying Technol., 2014, 32, 813-819.

7. Banwart G. 2012. Basic food microbiology: Springer Science \& Business Media, pp. 49-100.

8. Chandra S., Kumari D., Recent development in osmotic dehydration of fruit and vegetables: a review. Crit. Rev. Food Sci. Nutr., 2015, 55, 552-561.

9. Chong C.H., Law C.L., Figiel A., Wojdyło A., Oziembłowski M., Colour, phenolic content and antioxidant capacity of some fruits dehydrated by a combination of different methods. Food Chem., 2013, 141, 3889-3896.

10. Eliasson L., Libander P., Lövenklev M., Isaksson S., Ahrné L., Infrared decontamination of oregano: effects on Bacillus cereus spores, water activity, color, and volatile compounds. J. Food Sci., 2014, 79, E2447-E2455.

11. Ghosh D., Das S., Bagchi D., Smarta R., Innovation in healthy and functional foods, 2012 CRC, Press. pp 193.

12. Guiamba I.R., Svanberg U., Effects of blanching, acidification, or addition of EDTA on vitamin $\mathrm{C}$ and $\beta$-carotene stability during mango purée preparation. Food Sci. Nutr., 2016, 4, 706-715.

13. Holzwarth M., Wittig J., Carle R., Kammerer D.R., Influence of putative polyphenoloxidase (PPO) inhibitors on strawberry (Fragaria $\mathrm{x}$ ananassa Duch.) PPO, anthocyanin and color stability of stored purées. LWT Food Sci. Technol., 2013, 52, 116-122.

14. Hopping B.N., Erber E., Grandinetti A., Verheus M., Kolonel L.N., Maskarinec G., Dietary fiber, magnesium, and glycemic load alter risk of type 2 diabetes in a multiethnic cohort in Hawaii. The Journal of nutrition, 2010, 140, 68-74.

15. Ioannou I., Ghoul M., Prevention of enzymatic browning in fruit and vegetables. Eur. Scientific J., 2013, 9, 310-341.

16. Jeevitha G., Anto A., Chakkaravarthi A., Hebbar H., Application of Electromagnetic Radiations and Superheated Steam for Enzyme Inactivation in Green Bell Pepper. Journal of Food Processing and Preservation, 2015, 39, 784-792.

17. Korbel E., Attal E.-H., Grabulos J., Lluberas E., Durand N., Morel G., Goli T., Brat P., Impact of temperature and water activity on enzymatic and non-enzymatic reactions in reconstituted dried mango model system. Eur. Food Res. Technol., 2013, 237, 39-46.

18. Lemmens L., Tchuenche E.S., Van Loey A.M., Hendrickx M.E., Beta-carotene isomerisation in mango puree as influenced by thermal processing and high-pressure homogenisation. Eur. Food Res. Technol., 2013, 236, 155-163.

19. Meilgaard M.C., Carr B.T., Civille G.V. 2006. Sensory evaluation techniques: CRC press.

20. Munyaka A.W., Oey I., Van Loey A., Hendrickx M., Application of thermal inactivation of enzymes during vitamin $\mathrm{C}$ analysis to study the influence of acidification, crushing and blanching on vitamin $\mathrm{C}$ stability in Broccoli (Brassica oleracea L var. italica). Food Chem., 2010, 120, 591-598.

21. Qian C., Decker E.A., Xiao H., McClements D.J., Physical and chemical stability of $\beta$-carotene-enriched nanoemulsions: Influence of $\mathrm{pH}$, ionic strength, temperature, and emulsifier type. Food Chem., 2012, 132, 1221-1229. 
22. Ranganna S. 1986. Handbook of analysis and quality control for fruit and vegetable products: Tata McGraw-Hill Education.

23. Rincon A., Kerr W.L., Influence of osmotic dehydration, ripeness and frozen storage on physicochemical properties of mango. Journal of Food Processing and Preservation, 2010, 34, 887-903.

24. Ruiz-Ojeda L.M., Peñas F.J., Comparison study of conventional hot-water and microwave blanching on quality of green beans. Innovative Food Science \& Emerging Technologies, 2013, 20, 191-197.

25. Singh N.I., Dhuique-Mayer C, Lozano YVES. Physico-chemical changes during enzymatic liquefaction of mango pulp (cv. Keitt). Journal of Food Processing and Preservation, 2000, 24, 73-85.

26. Slavin J., Fiber and prebiotics: mechanisms and health benefits. Nutrients, 2013, 5, 1417-1435.

27. Slavin J.L., Lloyd B., Health benefits of fruits and vegetables. Adv. Nutr: An International Review Journal, 2012, 3, 506-516.
28. Sogi D., Siddiq M., Roidoung S., Dolan K., Total Phenolics, Carotenoids, Ascorbic Acid, and Antioxidant Properties of Fresh cut Mango (Mangifera indica L., cv. Tommy Atkin) as Affected by Infrared Heat Treatment. J. Food Sci., 2012, 77, C1197-C1202.

29. Sra S., Sandhu K., Ahluwalia P., Effect of treatments and packaging on the quality of dried carrot slices during storage. J. Food Sci. Technol., 2014, 51, 645-654.

30. Vishwanathan K.H., Giwari G.K., Hebbar H.U., Infrared assisted dry-blanching and hybrid drying of carrot. Food Bioprod. Process., 2013, 91, 89-94.

31. Yadav A.K., Singh S.V., Osmotic dehydration of fruits and vegetables: a review. J. Food Sci. Technol., 2014, 51, 1654-1673.

Submitted: 7 September 2016. Revised: 9 December 2016. Accepted: 25 January 2017. Published on-line: 12 July 2017. 
